\title{
ASPECTOS METODOLÓGICOS E DE UTILIZAÇÃO DO FATOR DE IMPACTO
}

\author{
Catia Cândida Almeida \\ Doutora em Ciência da Informação. \\ caticandida@gmail.com \\ https://orcid.org/0000-0002-8477-6257
}

\author{
Maria Cláudia CabriniGracio \\ Professora do Programa de Pós-Graduação em Ciência da \\ Informação da Universidade Estadual Paulista (UNESP). \\ https://orcid.org/0000-0002-8003-0386
}

\begin{abstract}
RESUMO
A presente pesquisa objetiva identificar os aspectos metodológicos e de utilização do Fator de Impacto, os quais são alvo de críticas e de questionamentos, por parte dos pesquisadores. Como procedimento metodológico, realizou-se um levantamento bibliográfico, a partir da base de dados Web of Science, sendo identificados nove artigos, nos períodos de 1995 a 2018. Como resultado, observou-se que as críticas apontadas se relacionam à composição dos periódicos, na base de dados Web of Science, que não é padronizada, em termos de classificação dos tipos de documentos; à representatividade das áreas científicas e à abrangência regional. Ainda, a diferença na distribuição das citações, entre as áreas, sem a devida normalização para a comparação entre elas, configurou importante crítica ao uso do Fator de Impacto. Considerando a contemporaneidade dos resultados obtidos, conclui-se que os problemas metodológicos e de uso do Fator de Impacto ainda constituem problemas atuais nas publicações científicas, que embasam seus resultados neste indicador.
\end{abstract}

Palavras-chave: Ciência da Informação. Produção Científica. Avaliação de Periódicos. Fator de Impacto.

\section{METHODOLOGICAL ASPECTS AND IMPACT FACTOR USE}

\begin{abstract}
This research aims to identify the methodological and use aspects of the Journal Impact Factor, which are the target of criticism and questioning by the researchers. As a methodological procedure, a bibliographic research was carried out from the Web of Science database, and nine articles were identified from 1995 to 2018. As result, it was observed that the criticisms related to the composition of the journals in the literature. Web of Science database, which is not standardized in terms of classification of document types; the representativeness of the scientific areas and the regional scope. Also, the difference in the distribution of citations between the areas, without proper normalization for the comparison between them, was an important criticism of the use of the Journal Impact Factor. Considering the contemporaneity of the results obtained, it is concluded that methodological and use problems of the Journal Impact Factor still constitute current problems in scientific publications that base their results on this indicator.
\end{abstract}

Keywords: Information Science. Scientific Production. Journal Evaluation. Journal Impact factor.

Recebido em: 19/11/2019 Aceito em: 20/08/2020 Publicado em: 11/09/2020

\section{INTRODUÇÃO}

A avaliação científica, no que se refere aos julgamentos e às decisões de um trabalho científico, visa contribuir para o avanço do conhecimento. Diversas fontes de informações, tais como, artigos e livros, entre outras, subsidiam as avaliações da produção dos cientistas, grupos, instituições e países. 
Nesse cenário, a Bibliometria desenvolve métodos quantitativos de mensuração da literatura, de documentos e de outros canais de comunicação científica,a fim de medir o estado da ciência e da tecnologia científica. É uma forma de situar a produção de um país em relação ao mundo, uma instituição de país e os cientistas às suas próprias comunidades. (SPINAK, 1998; MACIAS-CHAPULA, 1998). Ela tem um papel importante no desenvolvimento de indicadores quantitativos, que subsidiam as avaliações, agrupados em indicadores de produção, de citação e de ligação. (GLÄNZEL; MOED, 2013; SPINAK, 1998; OKUBO, 1997; WALTMAN, 2016).

Particularmente, em relação aos indicadores de citação ou indicadores de impacto de citação, o propósito é a mensuração da intensidade do impacto das publicações científicas, calculados a partir da contagem das citações. $O$ indicador mais conhecido de impacto dos periódicos é o Fator de Impacto.(Journal Impact Factor). (SPINAK, 1998; WALTMAN, 2016). Este foi desenvolvido por Eugene Garfield, do Institute for Scientific Information (ISI), com o propósito de selecionar revistas científicas da base de dados Science Citation Index (SCI), partindo da contagem de citação dos autores de uma revista, a fim de dar visibilidade ao impacto das revistas, com pequeno volume de artigos publicados, mas de grande relevância para o desenvolvimento da ciência. (GARFIELD, 1955, 1972; GARFIELD; SHER, 1963). Os dados de citação dos autores foram categorizados pelo ISI e passaram a ser usados como parâmetro de avaliação de pesquisadores e instituições. (STREHL, 2005)

Nesse contexto, o Fator de Impacto de um periódico é um indicador que retrata a frequência com que, em média, um artigo nele disseminado foi citado. Em termos matemáticos, foi definido como o quociente entre o número de citações recebidas no ano atual, relativo aos artigos publicados nos dois anos anteriores e o número total de documentos publicados nesses dois anos anteriores. Esse indicador consiste em um dos componentes do Jornal Citation Report (JCR), que fornece ferramentas quantitativas para classificar, avaliar, categorizar e comparar periódicos científicos. (CLARIVATE ANALYTICS, 2018).

Com o decorrer dos anos, o Fator de Impacto passou a ser utilizado de forma mais ampla, especialmente como sinônimo da qualidade das revistas.Os gestores da ciência começaram a empregá-lo como ferramenta de tomada de decisão, em questões de política científica, envolvendo pesquisadores individuais, grupos de pesquisa, 
departamentos, instituições e países, em lugar de seu uso limitar-se à avaliação de periódicos. (DE BELLIS, 2009).

Neste contexto, as agências de financiamento de pesquisa desenvolvem mecanismos de avaliação da produção científica dos pesquisadores e das instituições para a alocação de recursos financeiros. O Fator de Impacto vem ocupando um papel de destaque, como no caso da área da Medicina na Alemanha, em que esse indicador é utilizado para a alocação e distribuição dos recursos financeiros, entre os departamentos ligados à área. Este é um exemplo de experiência relatada dessa situação, o que passou a ser um problema complexo, por conta da heterogeneidade e epistemologia das disciplinas, tais como, genética molecular, cirurgia, medicina dentária, informática e médica, medicina social e outras áreas. (KALTENBORN; KUHN, 2003; KALTENBORN, 2004). Visto que análises comparativas de produções científicas entre áreas ou disciplinas não são recomendadas, pois, cada área ou disciplina tem um perfil de produção e impacto diferente,relacionando-se com a rapidez da comunicação científica e os tipos de canais utilizados na disseminação de suas pesquisas.

Por outro lado, as instituições educacionais e de pesquisa produzem rankings de classificação dos periódicos baseados no Fator de Impacto, a fim de avaliar o desempenho individual do pesquisador. Nesta averiguação, quanto maior o valor do Fator de Impacto dos periódicos presentes nos rankings de avaliação de uma instituição, maior a chance do candidato superar os outros em uma promoção, bolsa ou financiamento de projetos. Em alguns países europeus, é uma prática constante a associação do Fator de Impacto ao mérito individual do pesquisador. (BREMBS, 2018; DE BELLIS, 2009; PENNER et al., 2013; VARIN et. al., 2016).

Apesar do prestígio alcançado por esse indicador, especialmente junto às agências de fomentos, diversas críticas e limitações têm sido apresentadas na literatura, quanto a sua metodologia de construção e sua utilização na avaliação das pesquisas. (FERNANDES-LLIMÓS, 2003; GLÄNZEL; MOED, 2002; KALTENBORN, 2004; SEGLEN, 1997; SIMONS, 2008; WALTMAN, 2016).

Nesse sentido, a literatura científica contém muitos trabalhos com diversas críticas ao Fator de Impacto. Estas censuras, em função da amplitude desse universo dificultam a reunião de toda controvérsia relativa às suas limitações metodológicas e conceituais. Waltman (2016) afirma que não é possível fazer uma revisão completa da literatura, 
que cubra todas as publicações sobre o tema "indicadores de citação", especialmente as críticas e os questionamentos sobre o Fator de Impacto. O autor ressalta que o Fator de Impacto provoca debates e discussões, de diferentes pontos de vistas, na comunidade científica, envolvendo conceito, formulação matemática e, principalmente, a sua forma de utilização nas avaliações de pesquisas.

Nesta perspectiva, este trabalho identifica os artigos científicos que discutem questões associadas aos aspectos metodológicos e de utilização do Fator de Impacto, que têm sido alvo de críticas e questionamentos, por parte dos pesquisadores. Em uma perspectiva diacrônica, elegeram-se nove trabalhos publicados no período de 1995 a 2018, analisando as principais críticas aos procedimentos metodológicos e de utilização desse indicador. Este estudo foi motivado, a partir da ideia de investigação do entendimento do significado ou representação do Fator de Impacto para os pesquisadores da área da Ciência da Informação. Antes dessa investigação, surgiu a necessidade de se fazer um levantamento dos principais julgamentos referentes aos aspectos metodológicos e de utilização do Fator de Impacto.

Este trabalho se justifica, tomando como um exemplo de ambiente de utilização do Fator de Impacto, as instituições educacionais e de pesquisa, principalmente as brasileiras, que oferecem programas de pós-graduação. Estas são submetidas a processos avaliativos de órgãos de acompanhamento, avaliação e reguladores da ciência, entre eles a Coordenação de Aperfeiçoamento de Pessoal de Nível Superior (CAPES). Neste contexto, um dos instrumentos utilizados nessa avaliação foi o sistema Qualis-periódicos, que consiste na classificação de periódicos, dispondo do Fator de Impacto, com um dos indicadores mais utilizados como critério de qualificação de periódicos. (BARATA, 2016; CAMPOS, 2010; CAPES, 2019). Assim, vale destacar as principais críticas associadas aos aspectos metodológicos e de utilização desse indicador, que se faz presente no Qualis-periódicos e em avaliações científicas.

\section{METODOLOGIA}

Esta pesquisa é de natureza qualitativa e descritiva, baseada em um levantamento bibliográfico sobre o tema. É motivada, segundo o estudo de Van Leeuwen (2012), que apresentou algumas críticas a respeito do Fator de Impacto em blocos de assuntos associados aos aspectos matemáticos/numéricos e a aspectos metodológicos/utilização. 
Para o desenvolvimento dessa pesquisa, foram consideradas as críticas envolvendo os aspectos metodológicos e de utilização do Fator de Impacto. A identificação e seleção do corpus da pesquisa partiu da base de dados WoS, coleção principal, buscando-se os artigos que apresentassem o termo "Journal Impact Factor", em todos os campos, tipologia documental "artigo" e período de 1995 a 2018, totalizando um universo de 122.786 artigos. Diante desse imenso universo de artigos, optou-se por delimitar a pesquisa em uma linha temporal, escolhendo os anos de 1995 (903 artigos), 1996 (1.046 artigos) e 1997 (1.164 artigos), seguido de 2007 (3.915 artigos), 2008 (4.645 artigos) e 2009 (5.123 artigos) e os anos mais recentes 2016 (12.347 artigos), 2017 (13.072 artigos) e 2018 (14.374 artigos).

Em seguida, selecionou-se, de forma intencional, um artigo de cada ano, levando em consideração a quantidade de citação recebida e 0 assunto envolvendo críticas metodológicas e de utilização do Fator de Impacto. Assim, elegeram-se nove artigos, a saber: três trabalhos no final da década de 1990 - Moed e Van Leeuven (1995), Rousseau e Van Hooydonk (1996), Seglen (1997), seguido de três no final dos anos 2000 - AleixandreBenavent et al. (2007), Durand-Barthlez (2008), Cross (2009) e, por fim, três mais recentes Waltman (2016), Zhang, Rousseau e Sivertsen (2017) e Moed (2018). Ressalta-se que o propósito desse estudo foi apenas analisar as principais críticas e questionamentos, referentes aos aspectos metodológicos e de utilização do Fator de Impacto.

A visualização desses trabalhos é apresentada em uma linha temporal, na Figura 1, com os nomes dos autores e os anos de publicações dos trabalhos.

Figura 1 - Linha temporal com os autores e ano de publicação das principais críticas metodológicas e de utilização do Fator de Impacto.

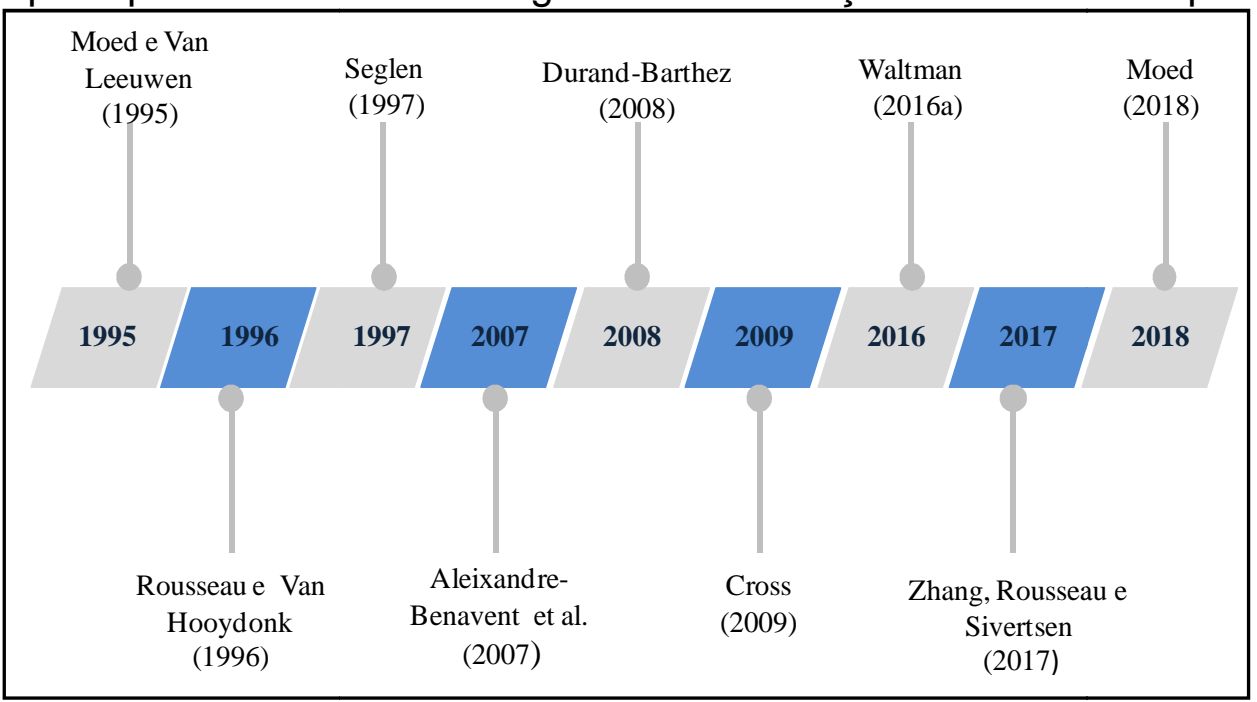

Fonte: Elaboração própria 
Por fim, esses trabalhos foram analisados e as principais críticas metodológicas e de utilização foram agrupadas nos Quadro 1, Quadro 2 e Quadro 3.

\section{RESULTADOS E DISCUSSÃO}

Os quadros apresentados como Quadro 1, Quadro 2 e Quadro 3 descrevem uma síntese dos tópicos tratados, em cada um dos artigos.

Quadro 1 - Autores e principais tópicos relacionados à metodologia e ao uso do Fator de Impacto, dos artigos publicados nos anos 1995, 1996 e 1997

\begin{tabular}{|c|c|}
\hline Autores & $\begin{array}{l}\text { Principais tópicos relacionados à metodologia } \\
\text { e ao uso do Fator de Impacto }\end{array}$ \\
\hline $\begin{array}{c}\text { Moed e van Leeuven } \\
\text { (1995) }\end{array}$ & $\begin{array}{l}\text { 1. Definição dos tipos de documentos: há evidências de que, para } \\
\text { um grupo de periódicos com altos valores de Fator de Impacto, } \\
\text { esses valores são imprecisos, devido à definição e classificação } \\
\text { inadequada dos tipos de documentos, uma vez que os } \\
\text { documentos classificados como não citáveis (cartas e editoriais) } \\
\text { são citados e contribuem para a contagem de citação. } \\
\text { 2. Proposta de mudança na metodologia do cálculo do Fator de } \\
\text { Impacto: já que é perceptível a influência do conjunto de } \\
\text { documentos incluídos na contagem de citação sobre os valores } \\
\text { de Fator de Impacto dos periódicos. } \\
\text { 3. Divergência entre as datas inseridas na base WoS: o ano de } \\
\text { inserção dos artigos dos periódicos, nas bases de dados, } \\
\text { influencia nos valores de Fator de Impacto, uma vez que o } \\
\text { intervalo de tempo entre a entrada do artigo no sistema e sua } \\
\text { publicação varia ao longo das edições dos periódicos. } \\
\text { 4. Período de citação: o período de citação de um ou dois anos } \\
\text { relaciona-se com a rapidez com que declina a obsolescência } \\
\text { dos artigos. } \\
\text { 5. Interpretação do Fator de Impacto: é o valor médio de uma } \\
\text { distribuição de citação assimétrica , sendo necessárias outras } \\
\text { estatísticas para essa distribuição. }\end{array}$ \\
\hline $\begin{array}{l}\text { Rousseau e Van Hooydonk } \\
\text { (1996) }\end{array}$ & $\begin{array}{l}\text { 1. Forte correlação negativa entre a quantidade de artigos } \\
\text { publicados anualmente e o Fator de Impacto dos periódicos: } \\
\text { os periódicos que publicam muitos artigos, por ano, tendem a ter } \\
\text { baixos valores de Fator de Impacto e vice-versa. } \\
\text { 2. Correlação moderada entre as disciplinas em que atuam os } \\
\text { periódicos e seus respectivos valores de Fator de Impacto. }\end{array}$ \\
\hline $\begin{array}{l}\text { Seglen } \\
(1997)\end{array}$ & $\begin{array}{l}\text { 1. Os valores de Fator de Impacto dos periódicos não são, } \\
\text { estatisticamente, representativos dos artigos individualmente. } \\
\text { 2. Os valores de Fator de Impacto dos periódicos têm correlação } \\
\text { fraca com as citações dos artigos individualmente. } \\
\text { 3. Os autores usam muitos critérios além do Fator de Impacto } \\
\text { quando pretendem submeter seus artigos. } \\
\text { 4. Citações de itens "não citáveis" são erroneamente incluídas } \\
\text { na base. }\end{array}$ \\
\hline
\end{tabular}




\begin{tabular}{|c|c|}
\hline Autores & $\begin{array}{l}\text { Principais tópicos relacionados à metodologia } \\
\text { e ao uso do Fator de Impacto }\end{array}$ \\
\hline $\begin{array}{l}\text { Seglen } \\
(1997)\end{array}$ & $\begin{array}{l}\text { 5. As autocitações não são corrigidas. } \\
\text { 6. Os artigos de revisão são fortemente citados e inflam os valores } \\
\text { de Fator de Impacto dos periódicos. } \\
\text { 7. Os artigos extensos obtêm muitas citações e contribuem para } \\
\text { altos valores de Fator de Impacto dos periódicos. } \\
\text { 8. O tempo de publicação de um artigo, estabelecido por alguns } \\
\text { periódicos, estimula os autores a publicarem materiais de } \\
\text { curto prazo e a autocitação. Com isso, aumenta o valor do } \\
\text { Fator de Impacto. } \\
\text { 9. As citações no idioma nativo do periódico são privilegiadas pelos } \\
\text { editores de alguns periódicos. } \\
\text { 10. Autocitação seletiva dos periódicos: os artigos tendem a citar } \\
\text { outros artigos do mesmo periódico. } \\
\text { 11. A cobertura da base de dados não é completa. } \\
\text { 12. Os livros não estão incluídos nas bases de dados como fonte } \\
\text { de citações. } \\
\text { 13. A base de dados tem uma distorção, favorecendo a língua inglesa. } \\
\text { 14. A base de dados é dominada por publicações americanas. } \\
\text { 15. O conjunto de periódicos, na base de dados, pode variar de ano } \\
\text { para ano. } \\
\text { 16.O valor de Fator de Impacto é uma função do número de } \\
\text { referências, por artigo, na área da pesquisa. } \\
\text { 17. Há áreas de pesquisa cuja literatura se torna, rapidamente, } \\
\text { obsoleta e que são favorecidas, em relação ao Fator de Impacto. } \\
\text { 18. O FI depende da dinâmica (expansão ou contração) da área } \\
\text { da pesquisa. } \\
\text { 19. Áreas de pesquisa pequenas tendem a não ter periódicos com } \\
\text { valor alto de Fator de Impacto. } \\
\text { 20. As relações entre as áreas (no caso de clínica e pesquisa básica) } \\
\text { determinam fortemente o valor de Fator de Impacto do periódico. } \\
\text { 21. As taxas de citação do artigo determinam o impacto do } \\
\text { periódico, mas esta não é uma relação inversa. }\end{array}$ \\
\hline
\end{tabular}

Fonte: Elaboração própria

Destacam-se os tópicos que envolvem problemas de classificação dos tipos de documentos, cobertura da base de dados WoS e padrões de citações de diferentes áreas científicas. Moed e Van Leeuven (1995) e Seglen (1997) apontam como uma das principais críticas que permeiam o Fator de Impacto dos periódicos na literatura a classificação em termos de tipos de documentos (cartas, comentários, resumos e materiais editorias, entre outros documentos) não padronizada, afetando, diretamente, no cálculo do valor do Fator de Impacto dos periódicos.

Neste sentido, de acordo com estudos de Moed e Van Leeuwen (1995), Van Leeuwen e Moed (2002) e Seglen (1997), a base de dados WoS não classifica 
os tipos de documentos de forma consistente, criando uma desigualdade entre o denominador e o numerador da formulação matemática. Com exemplo, os documentos, como artigos, notas e resenhas são contabilizados no denominador (fórmula de cálculo Fator de Impacto), enquanto que as cartas, comentários, resumos e materiais editorias contabilizadas no numerador (fórmula de cálculo do Fator de Impacto), que, mesmo em quantidades menores, afetam os valores de Fator de Impacto. Essa situação interfere na contagem da quantidade de citação e documentos, consequentemente, inflacionando os valores de Fator de Impacto dos periódicos.

Moed e Van Leeuwen (1996) ilustram a questão, tomando como exemplo o periódico "Journal Lancet". Mostram que, se as contagens das citações e dos documentos levassem em conta o mesmo conjunto de elementos para o numerador e para 0 denominador, esse periódico apresentaria o valor do $\mathrm{FI}$ de $43 \%$ mais baixo do que o listado no Journal Citation Report, no ano de 1992. Também realizaram estudos com outros periódicos das áreas de Química, Medicina, Farmacologia, Farmácia, Engenharias, em que observaram que os periódicos apresentaram valores de Fator de Impacto até 10\% menores que aqueles publicados no JRC. Assim, se os mesmos documentos fossem considerados no numerador e denominador, haveria uma padronização dos tipos considerados no cálculo do Fator de Impacto, o que influenciaria em todos os resultados dos valores de Fator de Impacto.

O outro tópico mencionado pelos autores é referente à cobertura dos periódicos e distribuição da composição da base de dados, que contribuem para a limitação da representatividade do Fator de Impacto dos periódicos, em diversas áreas do conhecimento. Uma vez que o Fator de Impacto de qualquer periódico tende a ser proporcional à sua cobertura na base de dados, esta depende da área de pesquisa. Isso quer dizer que os periódicos de uma área ou país, de pouca representatividade na base, apresentam baixos valores de Fator de Impacto. (SEGLEN, 1997). Considera-se que a base de dados WoS indexa, prioritariamente, periódicos com artigos na língua inglesa, países da Europa e EUA.Dessa forma, a avaliação da produção científica, no âmbito regional de países, como o caso dos periódicos brasileiros, muitos periódicos não estão indexados na WoS, a pesquisa brasileira tem pouca representatividade na WoS.

Nessa perspectiva, um dos problemas do Fator de Impacto diz respeito à abrangência regional, uma vez que há periódicos que não são contemplados nas bases 


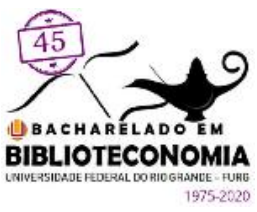

de dados, mas estão em outras bases. A título de exemplificação, cita-se o Instituto de Bases de dados de Informação Científica, da área de Ciências Sociais da Alemanha, com vários periódicos, ao passo que a base do ISI contempla uma quantidade menor de periódicos nessa área. (SEGLEN, 1997). Assim, considera-se significativo salientar que cada base de dados tem as suas políticas de indexação e que os pesquisadores precisam estar cientes dessa situação, no momento de realizar as suas pesquisas apoiadas nos indicadores bibliométricos.

O Quadro 2 apresenta os principais tópicos considerados pelos autores como relevantes aos conceitos metodológicos e de utilização do Fator de Impacto.

Quadro 2 - Autores e principais tópicos relacionados à metodologia e ao uso do FI, dos artigos publicados nos anos de 2007, 2008 e 2009

\begin{tabular}{|c|c|}
\hline Autores & Principais tópicos relacionados à metodologia e ao uso do FI \\
\hline $\begin{array}{l}\text { Aleixandre-Benavent et. al. } \\
\qquad(2007)\end{array}$ & $\begin{array}{l}\text { 1. Análise de citações: a função de citações na ciência moderna é } \\
\text { reconhecer que os métodos, interpretações, dados, etc., provêm } \\
\text { de autores e publicações citadas. Os trabalhos são citados não } \\
\text { apenas por sua influência, mas também por outras razões, dentre } \\
\text { as quais podem ser nomeações, argumentação ou para criticar } \\
\text { ou corrigi-los. Há outros fatores que devem ser levados em } \\
\text { consideração: áreas, temáticas, especialidades, escassez ou } \\
\text { ausência de trabalhos de qualidade em periódicos de países em } \\
\text { desenvolvimento, influência das barreiras linguísticas, baixa } \\
\text { divulgação de periódicos nas bibliotecas e base de dados. } \\
\text { 2. Base de dados WoS: um dos problemas mais graves nas bases é } \\
\text { a distorção da cobertura da literatura em favor dos periódicos } \\
\text { publicados na língua inglesa. Essa situação é uma desvantagem } \\
\text { para os países que não falam inglês. Dessa maneira, a seleção } \\
\text { dos periódicos dessa base não é representativa da produção } \\
\text { científica internacional e, assim, o fato de o periódico estar } \\
\text { incluído na base, permitirá que o valor de Fator de Impacto seja } \\
\text { maior que os outros periódicos que não estão na base. } \\
\text { 3. Fator de Impacto: é uma medida geral obtida a partir das citações } \\
\text { recebidas pelo periódico por um ano, mas nem todos os artigos } \\
\text { receberam o mesmo número de citações. Portanto, não é correto } \\
\text { atribuir a todos o mesmo valor de Fator de Impacto (alguns } \\
\text { estudos mostram que } 50 \% \text { do total das citações são destinadas a } \\
\text { apenas 15\% dos artigos). A contagem das citações não é } \\
\text { diferenciada por tipo de documento, como comentários, cartas e } \\
\text { outros materiais editoriais. } \\
\text { 4. O Fator de Impacto não deve ser aplicado para comparar } \\
\text { disciplinas diferentes, uma vez que cada uma delas tem sua } \\
\text { cultura em relação ao processo de citação. } \\
\text { 5. O Fator de Impacto favorece áreas altamente desenvolvidas ou } \\
\text { de grande número de pesquisadores em comparação a áreas } \\
\text { menos desenvolvidas e com menor número de pesquisadores. }\end{array}$ \\
\hline
\end{tabular}


Continuação

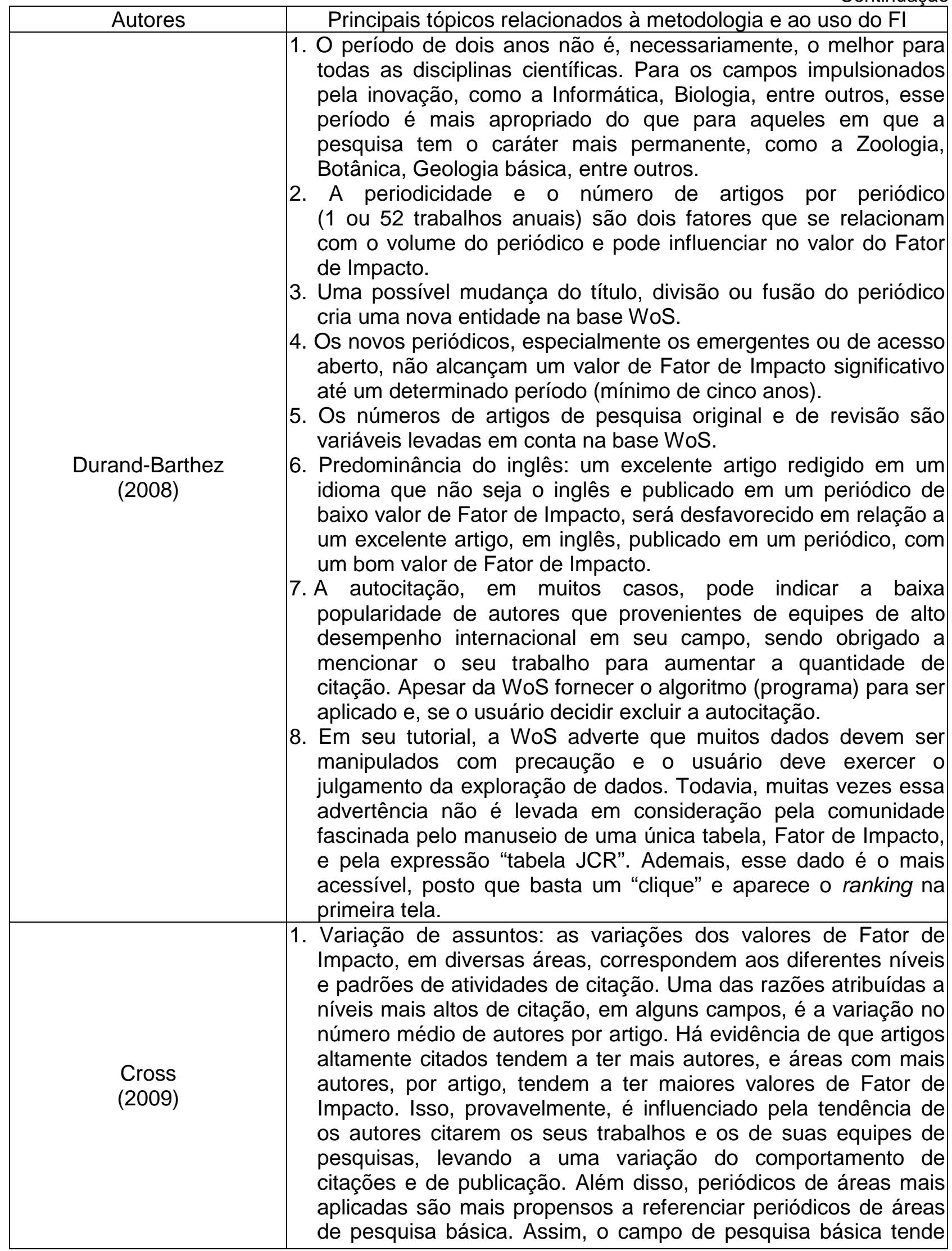


Continuação

\begin{tabular}{|c|c|}
\hline Autores & Principais tópicos relacionados à metodologia e ao uso do FI \\
\hline $\begin{array}{l}\text { Cross } \\
(2009)\end{array}$ & 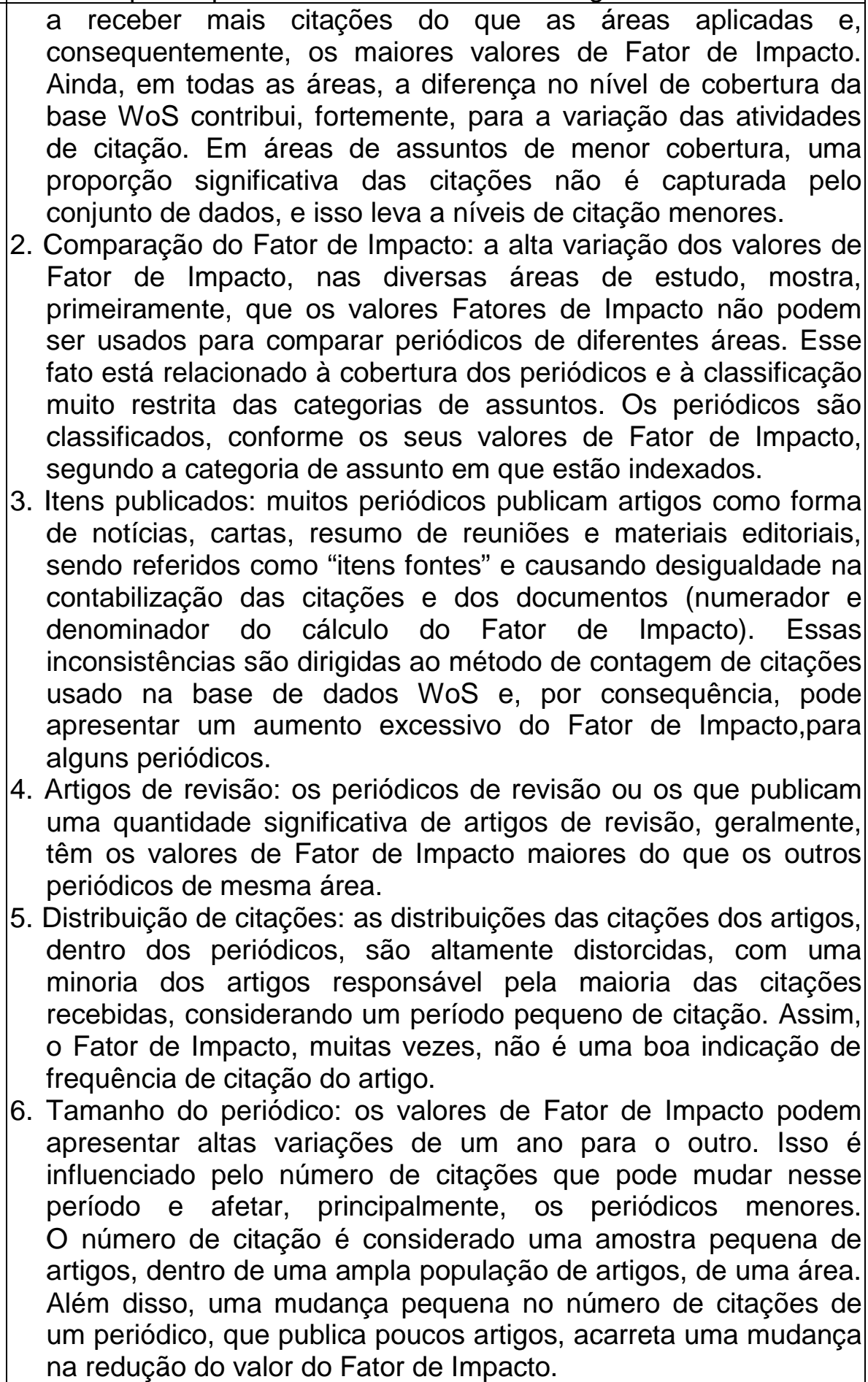 \\
\hline
\end{tabular}

Fonte: Elaboração própria

Destacam-se a contagem de citações, a distribuição de citação, a cobertura da base de dados WoS e os padrões de citação. A contagem de citações de um determinado período, ou seja, janela temporal de dois anos (atualmente, é a janela temporal 
considerada no cálculo do Fator de Impacto) após a publicação dos documentos, pode ter um efeito importante no cálculo do Fator de Impacto. Para exemplificar a dinâmica de pesquisa das áreas, existem publicações que crescem mais rapidamente, como no caso da "Biologia e Bioinformática", que tende a publicar trabalhos em um curto intervalo de tempo, e uma grande parcela desses documentos são citados no período de, aproximadamente, dois anos após sua publicação. Por outro lado, existem áreas em que as citações começam a aumentar após um período mais longo, como é o caso, também, das Ciências Sociais. (ALEIXANDRE-BENAVENT et al., 2007; CROSS, 2009; SEGLEN, 1997; WALTMAN, 2016).

Ademais, as citações e os valores de Fator de Impacto não são normalizados em relação às suas áreas, tornando-os absolutos, incomparáveis entre as áreas científicas. Por exemplo, o valor do Fator de Impacto dos periódicos de Biomedicina tende a ser maior do que aquele dos periódicos das Ciências Naturais, e cada vez mais, quando comparados aos valores de Fator de Impacto dos periódicos das Ciências Sociais. (CROSS, 2009; SEGLEN, 1997; VAN LEEUWEN; MOED, 1996).

Outro tópico, já mencionado neste estudo e destacado por esses autores, foi o fato de que os periódicos da base da WoS são predominantes de língua inglesa. Nesse cenário, os periódicos de língua não inglesa podem apresentar valores de Fator de Impacto, relativamente baixos, em decorrência da limitação de cobertura da base, visto que a maioria das citações, em outras línguas, é atribuída a documentos redigidos na mesma língua dos citantes. (DURAND-BARTHEZ, 2008; FERNANDÉZ-LLIMÓS, 2003). Em razão dessa limitação, os pesquisadores devem ficar atentos à cobertura da base de dados WoS e às políticas de indexação de periódicos. E, em contrapartida, desenvolver ferramentas de avaliação do impacto de periódicos de países periféricos.

Além disso, com o crescimento de publicações eletrônicas e as disponibilidades online dos artigos, consistem em outro problema relacionado às bases de dados que influencia no valor de Fator de Impacto. Nesse sentido, o estudo realizado com periódicos da MEDLINE, classificado em três agrupamentos, segundo o tipo de conteúdo do documento disponibilizado para o acesso aberto - texto completo, resumos e nenhum conteúdo - evidenciou que a disponibilidade on-line aumentou, efetivamente, o Fator de Impacto dos periódicos do grupo de texto completo. Essa constatação exemplifica o sucesso de várias iniciativas de acesso aberto (OA - open access), como a BioMed 
Central (BMC) e a Public Library of Science (PLoS) (DURAND-BARTHEZ, 2008; MURALI et al., 2004). Nesse cenário, ressalta-se a iniciativa brasileira de criação da base SciELO, comprometida com publicações de acesso aberto, que propicia maior visibilidade à produção científica brasileira e permite sua indexação em bases de dados internacionais. (FAPESP, 2016).

O Quadro 3 apresenta os principais tópicos, em um período mais recente, relevantes aos conceitos metodológicos e de utilização do Fator de Impacto.

Quadro 3 - Autores e principais tópicos relacionados à metodologia e ao uso do FI do ano de2016, 2017, 2018

\begin{tabular}{|l|l|}
\hline Autores & Principais tópicos relacionados à metodologia e ao uso do FI \\
\hline 1. Indicações básicas de avaliação do impacto de citações: existe \\
uma grande quantidade de literatura científica que aborda o Fator \\
de Impacto, mas uma boa parte não é tanto sobre este, mas a \\
maneira como é utilizado para fins de avaliação de pesquisa. \\
Em particular, as críticas são sobre a utilização em avaliação de \\
publicações individuais, com base no periódico em que eles \\
divulgam as suas pesquisas. \\
2. Período de citação de dois anos do Fator de Impacto: a \\
contagem de citações é referente às publicações dos dois anos \\
anteriores, sendo assim, um indicador diacrônico. Em lugar de \\
considerar as publicações em um período fixo de dois anos, o \\
Fator de Impacto deveria considerar as publicações no mesmo \\
período temporal, assim sendo, sincrônico. \\
3. Impacto de citações normalizado: nos valores de Fator de \\
Impacto dos periódicos, não são corrigidas as diferenças de \\
densidade de citações entre as áreas. Para resolver essa \\
limitação, um grande número de propostas está presente na \\
literatura. \\
4. No impacto de citações recursivas, o Fator de Impacto deveria \\
atribuir mais peso às citações de periódicos de alto impacto do \\
que às citações de periódicos de baixo impacto. Em geral, \\
os indicadores de citações recursivas atribuem um peso às \\
citações, dependendo do "item fonte". Assim, citações de origem \\
de um documento de alto impacto têm mais peso do que citações \\
de documentos de baixo impacto. \\
5 Impacto das citações de periódicos versus publicações \\
individuais: as avaliações das publicações, baseadas no FI dos \\
periódicos,são mais atraentes por estarem mais facilmente \\
disponíveis do que as estatísticas do número de vezes que as \\
publicações individuais foram citadas. Por isso, muitas vezes ele \\
serve como o substituto das estatísticas de publicações. \\
Recomendações: \\
(a) Não introduzir novos indicadores de impacto de citações, \\
a menos que se tenha claro o valor agregado em relação aos \\
indicadores existentes.
\end{tabular}


Continuação

\begin{tabular}{|c|c|}
\hline Autores & Principais tópicos relacionados à metodologia e ao uso do FI \\
\hline $\begin{array}{l}\text { Waltman } \\
(2016)\end{array}$ & $\begin{array}{l}\text { (b) Prestar atenção aos fundamentos teóricos dos indicadores } \\
\text { de impacto de citação. } \\
\text { (c) Prestar atenção na maneira como os indicadores de impacto } \\
\text { de citação estão sendo usados na prática. } \\
\text { (d) Explorar novas fontes de dados, a fim de obter medições } \\
\text { mais sofisticadas. }\end{array}$ \\
\hline $\begin{array}{c}\text { Zhang, Rousseau e Sivertsen } \\
\text { (2017) }\end{array}$ & $\begin{array}{l}\text { 1. A maioria dos dados de citações de periódicos apresentam } \\
\text { curvas assimétricas, por causa dos periódicos que são mais } \\
\text { citados entre os pesquisadores } \\
\text { 2. Existe correlação linear moderada entre as citações de um artigo } \\
\text { e o valor de Fator de Impacto.Não se espera alta correlação em } \\
\text { razão da assimetria da distribuição das citações dos periódicos. } \\
\text { 3. A correlação entre autoria individual de um artigo e o valor de } \\
\text { Fator de Impacto pode mudar, significativamente, de um autor } \\
\text { para o outro. } \\
\text { 4. Não existe correlação linear positiva, de forma consistente, } \\
\text { da citação de um artigo individual e o valor de Fator de Impacto } \\
\text { do periódico, no qual o artigo é publicado. } \\
\text { 5. As contribuições individuais de um trabalho não devem ser } \\
\text { avaliadas segundo o periódico que é usado para publicacãa. }\end{array}$ \\
\hline $\begin{array}{l}\text { Moed } \\
(2018)\end{array}$ & $\begin{array}{l}\text { 1. O uso de indicadores bibliométricos pode não levar ao aumento } \\
\text { de desempenho das pesquisas científicas. } \\
\text { 2. A qualidade de uma pesquisa é, cada vez mais, medida pela } \\
\text { quantidade de citações que recebe, mas seria necessário } \\
\text { desenvolver mais pesquisas empíricas para avaliar esses efeitos. } \\
\text { 3. Quando Garfield desenvolveu o Fator de Impacto, estava ciente } \\
\text { de que esse indicador seria utilizado para monitorar o } \\
\text { comportamento dos periódicos e dos editores. } \\
\text { 4. Garfield sempre enfatizou as limitações do uso do Fator de } \\
\text { Impacto dos periódicos para medir o desempenho individual dos } \\
\text { pesquisadores. } \\
\text { A avaliação individual do pesquisador, usando os dados } \\
\text { bibliométricos, deve ser realizada de forma minuciosa e } \\
\text { aproveitando as potencialidades das tecnologias da informação } \\
\text { e comunicação. Desse modo,desenvolveria métricas para } \\
\text { avaliação de um grupo que seria referência para servir de base } \\
\text { comparativa. }\end{array}$ \\
\hline
\end{tabular}

Fonte: Elaboração própria

Observa-se que, nesses tópicos mais recentes, os autores destacam as recomendações sobre a utilização do Fator de Impacto nas avaliações científicas. Waltman (2016), ainda, faz um alerta para a maneira de utilização dos indicadores de impacto de citação, em especial, o Fator de Impacto, muito utilizado em avaliações científicas, particularmente na avaliação de publicação individual do pesquisador. Zhang, Rousseau e Sivertsen (2017) analisaram os dados de citação dos periódicos e as contribuições individuais dos pesquisadores e concluíram que podem mudar, 
significativamente, de pesquisador para outro, independentemente do valor do Fator de Impacto do periódico que publicou o seu trabalho. Moed (2018), por fim, afirma que a avaliação individual do pesquisador, usando indicadores bibliométricos, deve ser realizada de forma minuciosa.

\section{CONSIDERAÇÕES FINAIS}

A síntese das descrições apresentadas nos artigos elucida os principais problemas metodológicos e de utilização do Fator de Impacto. Os três artigos analisados, em meados dos anos de 1990, trataram sobre os problemas metodológicos e de utilização envolvendo a classificação dos tipos de documentos, problemas de dados WoS, cobertura da WoS e padrões de citações, em diferentes áreas científicas.

Nos três artigos analisados, dos anos de 2007, 2008 e 2009, os autores colocam em foco a questão do período de dois anos do Fator de Impacto, comumente chamada de “janela de citação”. É considerado um período insuficiente para muitas áreas científica, particularmente às áreas de Ciências Sociais, muito embora, a Clarivate Analytics forneça o valor de Fator de Impacto, dos periódicos, em uma janela de cinco anos. Além disso, a cobertura da base de dados WoS tem baixa representatividade de periódicos de língua não inglesa. Os autores alertaram para o crescimento das publicações eletrônicas e disponibilidade on-line dos artigos que podem afetar o cálculo do Fator de Impacto dos periódicos, uma vez que os periódicos que tendem a ser mais citados, por conta da sua visibilidade e exposição. Por fim, considera-se a questão da diferença entre os padrões de citações entre os campos científicos do conhecimento, da contagem de citações e da distribuição das citações é praticamente unânime em todos os trabalhos.

Nos três artigos mais recentes, os autores tratam de questões ligadas à utilização do Fator de Impacto nas avaliações individuais de pesquisadores. São discutidas estas questões, de forma mais intensa, recomendando a não utilização do Fator de Impacto do periódico como um instrumento de avaliação individual do pesquisador.

No que diz a respeito às limitações desse estudo é importante salientar que se apresentou apenas os principais aspectos metodológicos e acerca da utilização do Fator de Impacto, com ênfase aos problemas ou pontos negativos, por isso se faz necessário reunir mais elementos na literatura que discutam outros problemas do Fator de Impacto. 
Isso porque, os autores podem não ter mencionados toda a problemática que envolve o Fator de Impacto.

Recomenda-se que a utilização do Fator de Impacto seja acompanhada de outros indicadores quantitativos e qualitativos. O impacto científico do periódico não pode ser sustentado por apenas um único indicador, já que se corre o risco de obter uma visualização parcial ou distorcida do comportamento científico.Sugere-se, portanto, o desenvolvimento de indicadores que retratem, de forma mais precisa, o impacto das pesquisas de países periféricos, com baixa representatividade na base de dados WoS, em especial, as pesquisas brasileiras. Aconselha-se, ainda, que o Fator de Impacto não deva ser utilizado de forma comparativa entre as áreas científicas, uma vez que cada área tem um perfil de produção e impacto diferente.

Para trabalhos futuros sugerem-se estudos envolvendo o Fator de Impacto e as críticas associadas aos periódicos de acesso aberto e acesso restrito e estudos que avaliem a relação entre a quantidade de citações de artigos publicados em periódicos com alto valores de Fator de Impacto e a qualidade desses artigos. Este estudo pode contribuir com as discussões detalhadas sobre a utilização do Fator de Impacto, no contexto da avaliação da produção científica, oriundas da área de Ciência da Informação, geralmente apontadas por outras áreas, como a Medicina, Química, Biomedicina entre outras. Além do mais, esta pesquisa reuniu as principais críticas apontadas na literatura, envolvendo os aspectos metodológicos e de utilização, em períodos distintos, com a intenção de evidenciar que as críticas ainda estão presentes na comunidade científica, em diferentes perspectivas.

\section{REFERÊNCIAS}

ALEIXANDRE-BENAVENTI, R.; MORENO-SOLANO, L. M.; SAPENA, F. A.; PEREZ, S. A. E. Correlation between impact factor and public availability of published research data in Information Science and Library Science journals. Scientometrics, Netherlands v. 106, p. 1-13, 2016. doi: 10.1007/s11192-016-1868-7.

BARATA, R. C. B. Dez coisas que você deveria saber sobre o Qualis. RBPG, Brasília, v. 13, n. 30, p. 013 - 040, jan./abr. 2016. Disponível em:

<ojs.rbpg.capes.gov.br/index.php/rbpg/article/download/947/pdf>. Acesso em: 20 nov. 2016.

BREMBS, B. Prestigious Science Journals Struggle to Reach Even Average Reliability. Frontiers Human Neuroscience, Switzerland, v. 12, p. 37, 2018. doi: 10.3389/fnhum.2018.00037.

CLARIVATE ANALYTICS. The Clarivate Analytics Impact Factor. 2018. Disponível em <https://clarivate.com/essays/impact-factor/>. Acesso em: 05 jan. 2018. 
CAMPOS, J. N. B. Qualis periódicos: conceitos e praticas nas Engenharias I. RBPG, Brasília, v. 7, n. 14, p. 477-503, dez. 2010. Disponível em:

<ojs.rbpg.capes.gov.br/Índex.php/rbpg/article/download/14/11>. Acesso em: 22 mar. 2018.

CAPES. 2019. Disponível em: <http://www.capes.gov.br/avaliacao/sobre-a-avaliacao>. Acesso em: 02 abr. 2019.

CROSS, J. Impact factors - the basics. The E-Resources Management Handbook, United kingdom, cap. 18, p. 1-12, 2009. doi: 10.1629/9552448-0-3.17.

DE BELLIS, N. Bibliometrics and Citation Analysis: from the Science Citation Index to Cybermetrics. Lanham, Maryland, Toronto, Plymouth, UK: The Scarecrow Press, 2009.

DURAND-BARTHEZ, M. Historique et critique dufacteur d'impact. Schedae, France, v. 7, n. 1, p. 67-76, 2008. Disponível em: <https://hal.archives-ouvertes.fr/hal-01356185/document>. Acesso em: 20 fev. 2018.

FAPESP. FUNDAÇÃO DE AMPARO À PESQUISA DO ESTADO DE SÃO PAULO. In: Indicadores de ciência; tecnologia e inovação em São Paulo. São Paulo, 2016.

FERNANDÉZ-LLIMÓS, F. S. Análisis de la cobertura del concepto de Pharmaceutical Care enfuentes primarias y secundarias de información. 2003. 266 f. Tesis. Granada: Universidad de Granada, Departamento de Farmacia y Tecnologia Farmacéutica, 2003.

GARFIELD, E. Citation indexes for science: A new dimension in documentation through association of ideas. Science, USA, v.122, n. 3159, p. 108-11, 1955. Disponível em <http://garfield.library.upenn.edu/papers/science1955.pdf> Acesso em: 10 dez. 2017.

GARFIELD, E. ; SHER, I. H. New Factors in the Evaluation of Scientific Literature Through Citation Indexing. American Documentation, USA, v. 4, n. 3, p. 195-201, 1963. Disponível em <http://garfield.library.upenn.edu/essays/v6p492y1983.pdf> Acesso em 11 nov. 2017.

GARFIELD, E. Citation analysis as a tool in journal evaluation. Science, USA, v. 17, p. 471-479, 1972a. Disponível em: <http://www.garfield.library. upenn.edu/essays/V1p527y196273.pdf>. Acesso em 11 nov. 2017.

GLÄNZEL, W.; MOED, F. H. Journal impact measures in bibliometric research. Scientometrics, Netherlands, v. 53, n. 2, p. 171-193, 2002. doi: 10.1023/A:1014848323806.

GLÄNZEL, W. ; MOED, F. H. Opinion paper: thoughts and facts on bibliometric indicators.

Scientometrics, Netherland, v. 96, n. 1, p. 381-394, 2013. doi: 10.1007/s11192-012-0898-z.

KALTENBORN, F. K. Validity and fairness of the impact factor. German: Birkhäuser, 2004.

KALTENBORN, F. K; KUHN, K. Der Impact-Faktorals Parameter zur Evaluation von

Forscherinnen/Forschern und Forschung. Med Klin, Germany, v. 98, n. 3, p. 153-169, 2003. doi: 10.1007/s00063-003-1240-6.

MACIAS-CHAPULA, C. A. O papel da informetria e da cienciometria e sua perspectiva nacional e internacional. Ciência da Informação, Brasilia, v. 27, n. 2, p. 134-40, maio/ago. 1998.

MOED, H; VAN LEEUWEN, T. Improving the Accuracy of Institute for Scientific Information's Journal Impact Factors. Journal of the American Society for information Science, USA, v. 46, n. 6, p. 461-467, 1995. Disponível em: <https://doi.org/10.1002/(SICl)10974571(199507)46:6<461:AID-ASI5>3.0CO;2-G>. Acesso em: 22 mar. 2017.

MOED, H.; VAN LEEUWEN, T. Impact factors can mislead. Nature, United Kingdom, v. 381, n. 186, 1996. Diponível em: <https://www.nature.com/articles/381186a0>. Acesso em: 20 abr. 2017.

MOED, H. F. Eugene Garfield's influences upon the Future of Evaluative Informetrics. Frontiers in Research Metrics and Analytics, Switzerland, v. 3, n. 5, p. 1-6, 2018. Disponível em: <https://www.frontiersin.org/articles/10.3389/frma.2018.00005/full>. Acesso em: 15 mar. 2019.

MURALI, N. S; MURALI, H. R; AUETHAVEKIA, T. P.; ERWIN, P. J.; MANDREKAR, J. N.; MANEK, 
N. J; GHOSH, A.K. Impact of FUTON and NAA bias on visibility of research. Mayo Clinic Porceedings, USA, v. 79, n. 8, p. 1001-1006, 2004. doi: 10.4065/79.8.1001.

OKUBO, Y. Bibliometric Indicators and analysis of research systems: methods and examples. In: STI Working Papers, 1997, Paris: OECD, 1997, 69 p.

PENNER, O; PAN, R. K.; PETERSEN, A. M; KASKI, K.; SANTO F. On the Predictability of Future Impact in Science Scientific Reports, United Kingdom, v. 3, n. 3052, 2013. doi: 10.1038/srep03052.

ROUSSEAU, R.; VAN HOOYDONK, G. Journal Production and Journal Impact Factors. Journal of the American Society for Information Science. USA, v. 47, n. 10, p. 775-780, 1996. doi: 10.1002/(SICI)1097-4571(199610)47:10<775::AID-ASI5>3.0.CO;2-\#.

SEGLEN, P.O. Citations and journal impact factors: Questionable indicators of research quality. Allergy, Germany, v. 52, v. 11, p. 1050-1056, 1997. doi: 10.1111/j.1398-9995.1997.tb00175.x. SIMONS, K. The misused Impact Factor. Science, USA, v. 322, n. 5899, p. 165, 2008. doi: 10.1126/science.1165316.

SPINAK, E. Indicadores cienciométricos. Brasília, Ciência da Informação, v. 27, n. 2, p. $141-148 ; 1998$.

STREHL, L. O fator de impacto do ISI e a avaliação da produção científica: aspectos conceituais e metodológicos. Ci. Inf., Brasília, v. 34, n. 1, p.19-27, jan./abr. 2005. Disponível em: <http://www.scielo.br/pdf/ci/v34n1/a03v34n1.pdf>. Acesso em 26 março 2016.

VARIN, C.; CATTELAN, M.; FIRTH, D. Statistical modelling of citation exchange between statistics journals. Journal Royal Statistical Society, United kingdom, n. 179, part 1, p. 1-63, 2016.

Disponível em: <https://rss.onlinelibrary.wiley.com/doi/epdf/10.1111/rssa.12124>. Acesso em 25 abril 2018.

WALTMAN, L. A review of the literature on citation impact indicators. Journal of Informetrics, Netherlands, v.10, n. 2, p.365-391, 2016. doi: 10.1016/j.joi.2016.02.007.

VAN LEEUWEN, T. Discussing some basic critique on Journal Impact Factors: revision of earlier comments. Scientometrics, Netherlands, v. 92, p. 443-455, 2012. doi: 10.1007/s11192-012-0677-x. ZHANG L., ROUSSEAU R., SIVERTSEN G. Science desserves to be judged by its contents, not by its wrapping: Revisiting Seglen's work on jornal impact and research evaluation. PLoS ONE, San Francisco, 2017. doi:10.1371/journal.pone.0174205. 